or sensitised patients would benefit from antifungal treatment. To aid treatment decisions and to monitor response more accurate methods to detect Aspergillus in sputum are needed. This study aimed to identify CF patients with Aspergillus colonisation, using real time PCR, and examine the relationship of colonisation to markers of sensitisation.

Methods 108 adult CF patients provided a sputum sample and a blood sample. Serological tests included total IgE, specific A. fumigatus IgE and specific A. fumigatus IgG performed by Phadia ImmunoCAP ${ }^{\circledR}$ assay, and $A$. fumigatus precipitins by counter immunoelectrophoresis. Sputum was homogenised with sputasol and sonication. $10 \mu \mathrm{l}$ was cultured on sabouraud agar (Oxoid, UK) for $72 \mathrm{~h}$. The remaining sample was used in a commercial real time PCR assay, MycAssay Aspergillus. Patients on antifungal treatment were excluded from serological data analysis.

Results $30 \%$ of the 108 sputum samples were positive for Aspergillus species by standard culture whereas $80 \%$ were positive for Aspergillus species by PCR. 15 patients were on antifungal therapy of whom 7 were PCR positive. Of the serological tests, only specific IgG correlated to positive PCR. Using a ROC curve, a specific IgG level above $65 \mathrm{mg} / \mathrm{l}$ gave $85 \%$ sensitivity and 100\% specificity for positive PCR. 12 patients met the 2003 consensus minimum criteria for ABPA. All were PCR positive supporting the use of antifungals for ABPA. 38 patients were sensitised to aspergillus (specific $\operatorname{IgE}>0.4$ $\mathrm{KUa} / \mathrm{l}$ ), 28 of these were PCR positive. A group of 32 patients was identified that had a rise in specific IgG and positive PCR but no $\operatorname{IgE}$ rise. They may represent 'aspergillus bronchitis'. All patients with negative serology were PCR negative.

Conclusion Real time PCR can accurately identify CF patients with Aspergillus in their sputum, including those in whom antifungal therapy is inadequate. However, PCR alone cannot distinguish between ABPA, sensitisation and colonisation. Positive PCR correlates to a specific IgG $>65 \mathrm{mg} / \mathrm{l}$. A randomised trial of antifungal therapy is required to determine if there is clinical benefit in treating PCR positive patients.

\section{S20 THE BACTERIAL CYTOSKELETON-A NEW ANTIMICROBIAL TARGET IN CYSTIC FIBROSIS PATHOGENS?}

doi:10.1136/thx.2010.150912.20

${ }^{1} \mathrm{~S}$ C Carnell, ${ }^{2} \mathrm{~J}$ D Perry, ${ }^{1} \mathrm{C}$ M A Khan, ${ }^{3}$ A De Soyza. Institute for Cell \& Molecular Biosciences, University of Newcastle, Newcastle-upon-Tyne, UK; ${ }^{2}$ Department of Microbiology, The Freeman Hospital, Newcastle-upon-Tyne, UK; ${ }^{3}$ Institute of Cellular Medicine, University of Newcastle, Newcastle-upon-Tyne, UK

Background Burkholderia cepacia complex (BCC) bacteria are opportunistic pathogens which cause severe lung infections in cystic fibrosis (CF) patients. Treatment of BCC infections is difficult due to the inherent multidrug resistance of BCC. There is a pressing need to find new bacterial targets for antimicrobials that provide functions essential for cell growth \& replication. A major component of the bacterial cytoskeleton is the actin homologue MreB. MreB maintains bacterial cell shape by forming filaments under the bacterial inner membrane. A22 is a cell permeable compound that disrupts MreB, destabilising the bacterial cytoskeleton and altering the bacterial shape.

Aims To investigate the MreB bacterial cytoskeleton as a novel target for antimicrobials.

Methods We have tested a synthetic library of A22-related compounds and identified compound Q22 as a potential antimicrobial of interest against BCC and Pseudomonas aeruginosa strains. BCC bacteria have been grown in the presence of $\mathrm{Q} 22$ and a number of phenotypic changes observed.
Results Q22 inhibited growth of all 9 BCC species tested, including $B$. cenocepacia. A reduction in growth rate and cell morphology changes were also observed (Abstract S20 Figure 1). Higher concentrations of $\mathrm{Q} 22$ were required to exert $B$. cenocepacia growth effects $(30 \mu \mathrm{g} / \mathrm{ml} \mathrm{Q} 22)$ when compared to P. aeruginosa (3 $\mu \mathrm{g} / \mathrm{ml} \mathrm{Q} 22)$, probably due to the presence of two mreB genes in the $B$. cenocepacia genome. BCC bacteria lipopolysaccharide (LPS) is known to play an important role during infection. We analysed the LPS profile of BCC bacteria grown in the presence of Q22 and selected strains show profile differences when compared to untreated bacteria. The influence of $\mathrm{Q} 22$ treatment on bacterial motility and Type 3 secretion, a virulence associated secretion system, was assessed. However, growth inhibition masked motility analysis and differences observed in secreted protein profiles could not be attributed to Type 3 secretion. The growth conditions required for induction of Type 3 secretion in vitro remain undefined.
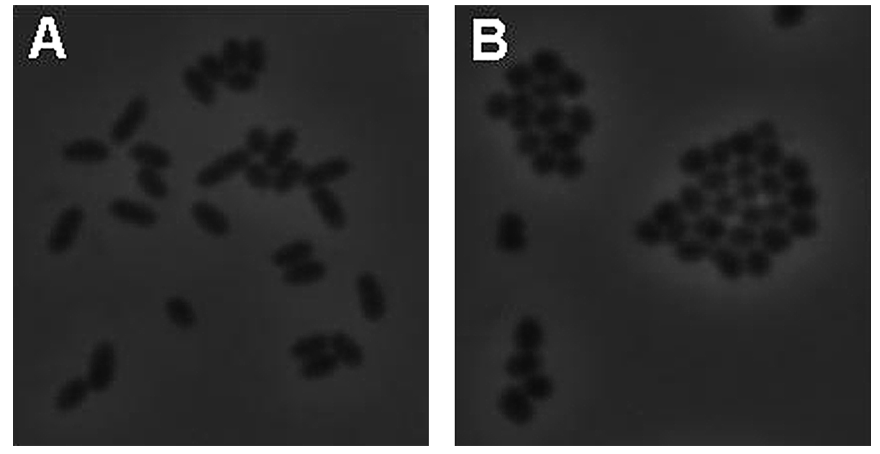

A, B. cenocepacia J2315 grown in LB with no additives;

\section{$\mathrm{B}, \mathrm{B}$. cenocepacia $\mathrm{J} 2315$ grown in LB containing 30ug/ml Q22}

\section{Abstract S20 Figure 1}

Conclusion In vitro $\mathrm{MreB}$ is an attractive new target for novel antimicrobials. Further analysis of current observations and additional phenotypic analysis will be required to dissect the nature of Q22induced changes. Work supported by Newcastle-Upon-Tyne Hospitals Special Trustees and Italian CF Research Foundation (FFC).

\section{S21 IDENTIFICATION OF PSEUDOMONAS AERUGINOSA INFECTION VIA VOLATILE ORGANIC COMPOUNDS IN SPUTUM HEADSPACE GASES}

doi:10.1136/thx.2010.150912.21

${ }^{1} \mathrm{~S}$ Savelev, ${ }^{2} \mathrm{~J}$ Perry, ${ }^{3} \mathrm{~S} J$ Bourke, ${ }^{1} \mathrm{R}$ Taylor, ${ }^{1} \mathrm{~A} J$ Fisher, ${ }^{4} \mathrm{M}$ Petrie, ${ }^{1} \mathrm{P}$ A Corris, ${ }^{1} \mathrm{~A}$ De Soyza. ${ }^{1}$ Newcastle University, Institute Cellular Medicine, Newcastle upon Tyne, UK; ${ }^{2}$ Dept Medical Microbiology Freeman Hospital, Newcastle upon Tyne, UK; ${ }^{3}$ Cystic Fibrosis Unit, Royal Victoria Hospital, Newcastle upon Tyne, UK; ${ }^{4}$ Newcastle University, Institute of Ageing \& Health, Newcastle upon Tyne, UK

Background Pseudomonas aeruginosa is a key respiratory pathogen with a distinctive odour in culture. An elevated level of hydrogen cyanide in the breath has been associated with the presence of $P$. aeruginosa in the airway, thus determining compounds specific to $P$. aeruginosa offers the possibility of a non-invasive diagnostic (breath) test.

Hypothesis Determining relevant to $P$. aeruginosa volatile compounds from sputum headspace gases offers target validation for the development of an electronic nose breath test for $P$. aeruginosa. 